\title{
Die Drohung der ungewissen Zukunft \\ Der Tod Nassers und Khomeinis als Epochenbruch
}

\author{
Johanna Pink und Olmo Gölz
}

Millionen Menschen mit schmerzverzerrtem Gesicht, Hunderttausende öffentlich weinend, Hunderte ohnmächtig, Trauer im Grenzbereich zur Massenphrenesie das hat die Welt bislang nur einmal erlebt: Nach dem Tod von Gamal Abd el-Nasser. ${ }^{1}$

\section{Einleitung}

Die Begräbnisse von Ğamāl 'Abd an-Nāșir (im Folgenden: Nasser) in Ägypten 1970 und von Âyatollāh Rūhollāh Homeinì (im Folgenden: Khomeini) in Iran 1989 gerieten zu Massenveranstaltungen historischen Ausmaßes, die Millionen in Kairo und Teheran mobilisierten und zu den größten Menschenansammlungen überhaupt zu zählen sind. Es ist nicht zuletzt der Wucht dieser Ereignisse und der jeweils aus ihnen entstandenen Bilder geschuldet, dass der Status der Verstorbenen als Nationalhelden nicht lediglich in ihren Begräbnissen bestätigt wurde, sondern postum aufrechterhalten, verfestigt oder gar noch gesteigert wurde - ein Prozess, der in beiden Fällen keinesfalls erwartbar war. Schließlich hatten sowohl Nasser als auch Khomeini in ihren letzten Lebensjahren im Angesicht zahlreicher Krisen erheblich an Zustimmung eingebüßt, sodass ihr politisches Charisma zumindest in Frage stand. In Iran spielte man gar mit dem Gedanken, Khomeini nachts zu beerdigen, damit die zu erwartende geringe Zahl der Trauernden sich nicht delegitimierend auf seinen Nachfolger auswirken würde. ${ }^{2}$ Die Ereignisse jedoch, die sich rund um die Leichname der beiden Nationalhelden entwickelten, schienen wie in einer affektiven Eruption ein letztes Mal die Außeralltäglichkeit Nassers und Khomeinis zu bestätigen, sodass die Episoden der Beerdigungen jeweils den heroischen Status der Protagonisten über ihren Tod hinaus maßgeblich konstituierten.

Nicht nur aufgrund der schieren Zahl der Menschen, die jeweils an den Beisetzungen der beiden Staatsmänner teilhaben wollten, weisen die Ereignisse auffallende Parallelen auf, welche weit über jenes $\mathrm{Ma}$ an Vergleichbarkeit hinausreichen, das aufgrund der politisch-biografischen Ähnlichkeiten der Verstorbenen zu erwarten gewesen wäre. Die Leistungen der beiden Protagonisten zu ihren Lebzeiten wurden dabei bisher insbesondere aufgrund von zwei Phänomenen vergli-

1 Der Spiegel: Nasser. Es kann nicht sein, 5. Oktober 1970, S. 130.

2 Vgl. Baqer Moin: Khomeini. Life of the Ayatollah, London 1999, S. 304. 
chen: Zum einen werden beide Akteure als Revolutionäre begriffen, deren Wirken nachhaltige Veränderungen im Staatensystem des Nahen und Mittleren Osten zur Folge hatte. ${ }^{3}$ Zum anderen werden sowohl Nasser als auch Khomeini mit populistischen Bewegungen assoziiert, die sich unmittelbar an ihren Personen selbst orientierten und gar als Nasserismus respektive Khomeinismus bezeichnet wurden. ${ }^{4}$ Eine Betrachtung der Beerdigungen der beiden Nationalhelden bringt ein weiteres Vergleichsmoment zutage: die emotionale Dimension beider Beisetzungen, deren Ausmaß eine Erklärung erfordert. Diese Erklärung wiederum eröffnet den Blick auf grundsätzliche Merkmale von Prozessen der Heroisierung.

Sowohl in Kairo 1970 als auch in Teheran 1989 kam es zu spontanen, nicht inszenierten Ansammlungen von Menschenmassen, die ihresgleichen suchen und die den Ablauf der Ereignisse entscheidend prägten. Die Menschenmengen waren hoch emotionalisiert bis hin zum Ausbruch von Massenhysterien mit etlichen Toten. So schrieb etwa ein Biograph Khomeinis über den 6. Juni 1989 in Teheran: „Words were inadequate to describe the event. ,Sheer frenzy', ,orgy of emotion', ,emotional orgasm' and a host of other phrases were used, but none could really describe the occasion. ${ }^{\text {"5 }}$ Diese Worte hätten auch über die Beerdigung Nassers in Kairo geschrieben werden können, wo die Massen das Ereignis an sich rissen und jegliche Organisation ad absurdum führten, was schließlich den Abbruch des vorgesehenen Ablaufs erzwang. In beiden Fällen schien der Drang danach, in die Nähe der Leiche zu gelangen, sie zu berühren und sich ihrer zu bemächtigen, übermächtig und prägte die Ereignisse maßgeblich.

Dieser Beitrag geht der Frage nach den Ursachen dieser Gemeinsamkeiten nach. Welche Eigenschaften und welche symbolischen Funktionen dieser beiden unzweifelhaft außeralltäglichen, heroischen Figuren und ihres Todes führte zu solch einer massenhaften Entladung von Emotionen im Angesicht ihrer Leichen? Welches Verhältnis zwischen Helden und ihren Verehrern manifestiert sich hier? Die Ereignisse um die Begräbnisse Nassers und Khomeinis sind mit dem Verweis auf den herausgehobenen Status der Verstorbenen, auf ihre jeweilige Rolle als Revolutionär oder Staatenlenker oder als charismatisches oder spirituelles Vorbild jedenfalls kaum erklärbar. Vielmehr verdichtet sich in den Prozessen um die Beerdigungen der beiden Nationalhelden und Identifikationsfiguren eine Symbo-

3 Vgl. Maridi Nahas: State Systems and Revolutionary Challenge. Nasser, Khomeini and the Middle East, in: International Journal of Middle East Studies 17, 1985, S. 507; Raymond A. Hinnebusch: Failed Regional Hegemons. The Case of the Middle East's Regional Powers, in: Seton Hall Journal of Diplomacy and International Relations 75, 2013, S. 78-82.

4 Vgl. Ervand Abrahamian: Khomeinism. Essays on the Islamic Republic, Berkeley/Los Angeles 1993, S. 2; Julia Kaspari / Rüdiger Robert: Identität durch Personenkult. Gamal Abdel Nasser und Ruhollah Khomeini, in: Rüdiger Robert u. a. (Hg.): Kollektive Identitäten im Nahen und Mittleren Osten. Studien zum Verhältnis von Staat und Religion, Münster 2010; Roy Mottahedeh: Iran's Foreign Devils, in: Foreign Policy 38, 1980, S. 33; Elie Podeh / Onn Winckler: Introduction. Nasserism as a Form of Populism, in: dies. (Hg.): Rethinking Nasserism. Revolution and Historical Memory in Modern Egypt, Gainesville 2004.

Moin: Khomeini (Anm. 2), S. 310-311. 
lik, die darauf hindeutet, dass mit dem Tod der jeweiligen Person die Wahrnehmung eines Epochenbruchs verbunden ist.

Einerseits scheinen sowohl Nasser als auch Khomeini von den Bevölkerungen Ägyptens und Irans als Repräsentanten oder gar explizit personifizierte Manifestation der jüngeren Nationalgeschichten selbst wahrgenommen worden zu sein, sodass sie den Menschen als jene Helden erschienen, die Hegel unter dem Begriff der ,welthistorischen Individuen' als Protagonisten der Geschichte beschreibt, „deren Taten einen Zustand und Weltverhältnisse hervorgebracht haben, welche nur ibre Sache und ibr Werk zu sein scheinen".6 Andererseits erwuchs mit dem Tod dieser beiden Repräsentanten der Geschichte eine Unsicherheit darüber, ob mit ihnen auch ihr ,Werk', das heißt, die von ihnen geformte Nation, in eine neue Zeit überführt werden müsse.

Die folgende knappe Darstellung der Begräbnisse Nassers und Khomeinis soll den Boden für eine Diskussion der Symbolebenen liefern, die sich auf die Wahrnehmung des Todes der beiden historischen Protagonisten als Epochenbruch konzentriert.

\section{Nasser}

Nasser war spätestens seit der Suezkrise von 1956 der Held der arabischen Welt. Sein diplomatischer - wenn auch nicht militärischer - Sieg über die ehemaligen Kolonialmächte Großbritannien und Frankreich sowie seine erfolgreiche Nationalisierung des Suezkanals verkörperten alle Hoffnungen, die die Bevölkerung der dekolonisierten Staaten und derjenigen, die noch um ihre Unabhängigkeit rangen, in diese Unabhängigkeit und ihre Führer setzten. Nasser hatte ein Ultimatum seitens der erdrückend überlegenen Allianz seiner Gegner nicht mit Verhandlungen oder einem Rücktritt beantwortet, sondern mit dem Versprechen, er und sein Volk würden bis zum letzten Blutstropfen kämpfen. ${ }^{7}$ Seine explizite Einordnung nicht nur als Führer und Kämpfer für die Unterdrückten, sondern als Held (batal) war in den ägyptischen Medien und denen vieler anderer arabischer Staaten allgegenwärtig. Der syrische Dichter Nizār Qabbānī (1923-1998) ließ einen fiktiven Soldaten aus dem Suezkrieg schreiben:

Dieser Brief, Vater, ist aus Port Said

wo Heldentum mit Blut und Stahl verschmilzt

aus dem Ort, der Helden produziert, schreibe ich dir, Vater aus Port Said...8

6 Georg Wilhelm Friedrich Hegel: Vorlesungen über die Philosophie der Geschichte, Frankfurt am Main ${ }^{11} 2015$, S. 46. Hervorhebungen im Original.

7 Vgl. Laura M. James: Nasser at War. Arab Images of the Enemy, Basingstoke 2006, S. 43.

8 Nizār Qabbānī: Al-a'māl as-siyāsiyya al-kāmila, Bd. 3, Beirut ${ }^{6} 2000$, S. 48. Soweit nicht anders angegeben, stammen die deutschen Übersetzungen von den Verfassern. 


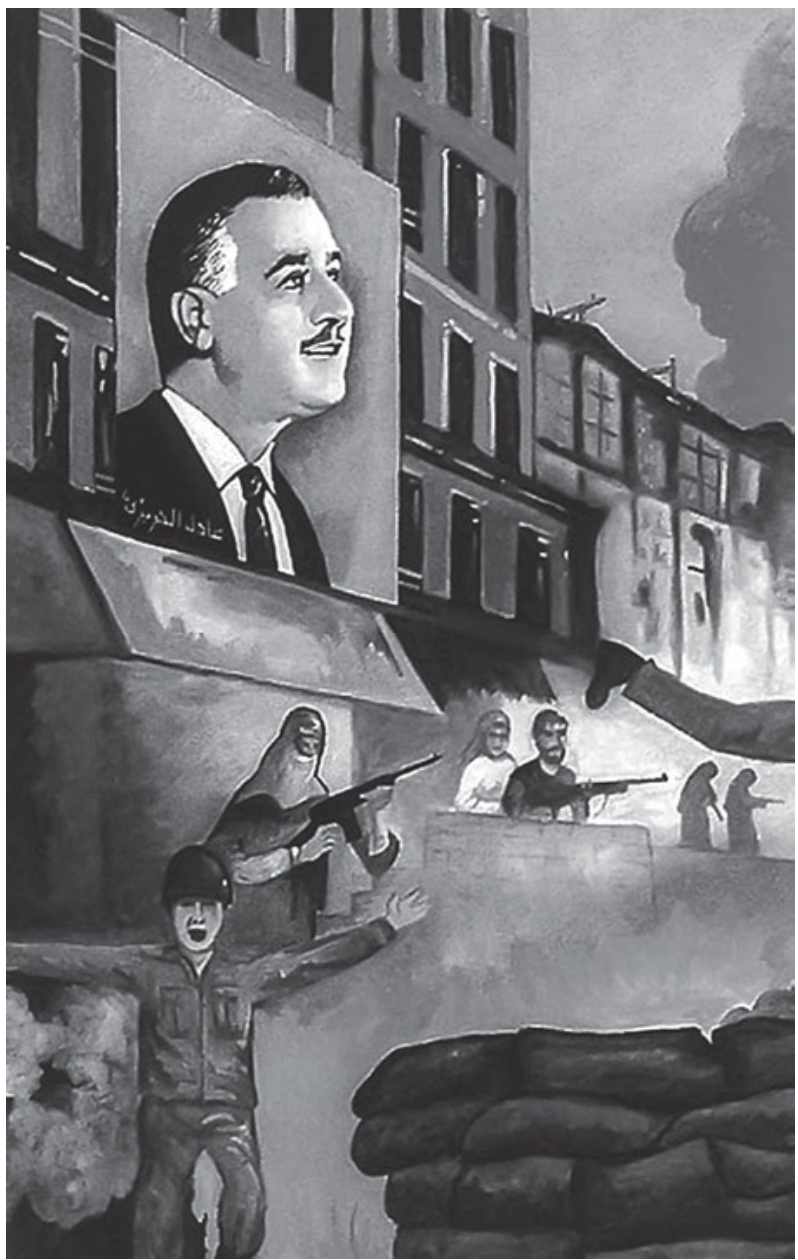

Abb. 1: Wandbild Nassers in Port Said, 2007.

In Port Said hatte die ägyptische Armee eine Niederlage erlitten und weite Teile der Stadt waren zerstört worden; doch durch Nassers diplomatischen Erfolg gingen die dort eingesetzten Soldaten, die Bevölkerung der Stadt sowie er selbst als Helden aus dem Krieg hervor (Abb. 1). ${ }^{9}$ Nassers Heldenstatus kam in Auftritten zum Ausdruck, in denen er von Volksmassen frenetisch umjubelt wurde. Der repressive Charakter seiner Herrschaft trat gegenüber dem von ihm verkörperten Versprechen auf Fortschritt, Stärke und Wohlstand in den Hintergrund; auch Misserfolge wie etwa das Scheitern der Union mit Syrien 1961 konnten seinem ikonischen Status zunächst nichts anhaben. ${ }^{10}$ Erst die verheerende Niederlage im

9 Vgl. Mériam N. Belli: An Incurable Past. Nasser's Egypt Then and Now, Gainesville 2013, S. $105-135$.

10 Vgl. Panayiotis J. Vatikiotis: Nasser and His Generation, London 1978, S. 319. 
Sechs-Tage-Krieg von 1967 stürzte ihn vom Sockel und zerstörte den Mythos der Unbesiegbarkeit. Doch selbst nach dieser Niederlage strömten Millionen von Ägyptern spontan auf die Straßen, um seinen Rücktritt zu verhindern und Nasser, wenn auch als gebrochenen und tragischen Helden, an der Macht zu halten: „He would stay in power not as a confident, vibrant hero, but as a tragic figure, a symbol of better days, an indication of the will to resist." ${ }^{11}$

Die Szenen, die sich am 28. September 1970 abspielten, als Nasser überraschend einem Herzinfarkt erlag, waren ein Echo derer von 1967 und Vorboten von Nassers Beisetzung am 1. Oktober: Ein Großteil der Bevölkerung der ägyptischen Großstädte strömte auf die Straßen, weinend und öffentlich trauernd, und skandierte: „Abū Huālid [Nasser], du lebst!“"12 Nassers Stellvertreter Anwar asSādāt hatte, als er unter Tränen den Tod des Präsidenten im Radio und Fernsehen bekanntgab, kommentiert, Nasser sei auf dem Schlachtfeld gestorben, im Kampf für die Einheit der arabischen Nation und den Tag des Sieges. Es sei nun die Pflicht der gesamten arabischen Nation, heroisch und standhaft zu bleiben, „damit sie den Sieg verwirklichen könne, für den der große Sohn Ägyptens, der Held dieser Nation, [...] gelebt hatte und den Märtyrertod starb." 13 Auch in anderen Ländern des Nahen Ostens kam es zu spontanen Trauerkundgebungen. Hunderttausende versammelten sich vor dem Präsidentenpalast, in dem Nassers Leiche aufgebahrt lag. ${ }^{14}$

Die Beerdigung Nassers drei Tage nach seinem Tod löste die vermutlich bis dahin größte Menschenansammlung, sicher aber den größten Trauerzug der ägyptischen Geschichte aus. Nach zeitgenössischen Schätzungen waren mindestens fünf, vielleicht sechs Millionen Ägypterinnen und Ägypter anwesend bei einer Gesamtbevölkerungszahl von ca. 35 Millionen; viele von ihnen waren aus anderen Teilen des Landes nach Kairo geströmt. Jedes Dach, jeder Baum und Laternenpfahl an der Prozessionsroute war voll mit Menschen. Die Planung sah einen würdevollen Staatsakt mit militärischen Ehren vor, doch die Soldaten und anwesenden Staatsgäste wurden nach kürzester Zeit abgedrängt von den Massen trauernder Menschen, die zum Sarg drängten und versuchten, ihn zu berühren und zu küssen (Abb. 2). Als die Trauernden versuchten, sich des Sarges zu bemächtigen, kam es zu gewaltsamen Auseinandersetzungen und einer Massenhysterie. Angesichts des vollständigen Verlusts der Kontrolle durch die Behörden wurde der Trauermarsch drei Stunden vor dem vorgesehenen Ende abgebrochen und der Sarg mit einem Militärfahrzeug zu der als letzte Ruhestätte vorgesehenen Moschee gefahren. Die Zahl der Toten soll sich auf 48 oder auch 143 Personen belaufen haben, die über-

11 Fouad Ajami: The Arab Predicament. Arab Political Thought and Practice since 1967, London 1981, S. 85. Vgl. auch Robert Stephens: Nasser, London 1971, S. 560, der die ägyptische Erinnerung an Nasser mit der französischen Erinnerung an Napoleon vergleicht.

12 Vgl. Jean Lacouture: Nasser, Paris 1971, S. 299-300; Stephens: Nasser (Anm. 11), S. 557; Saïd K. Aburish: Nasser. The Last Arab, New York 2004, S. 317.

13 Dan Hofstadter: Egypt \& Nasser. Volume 3: 1967-1972, New York 1973, S. 259.

14 Vgl. ebd., S. 259-260. 


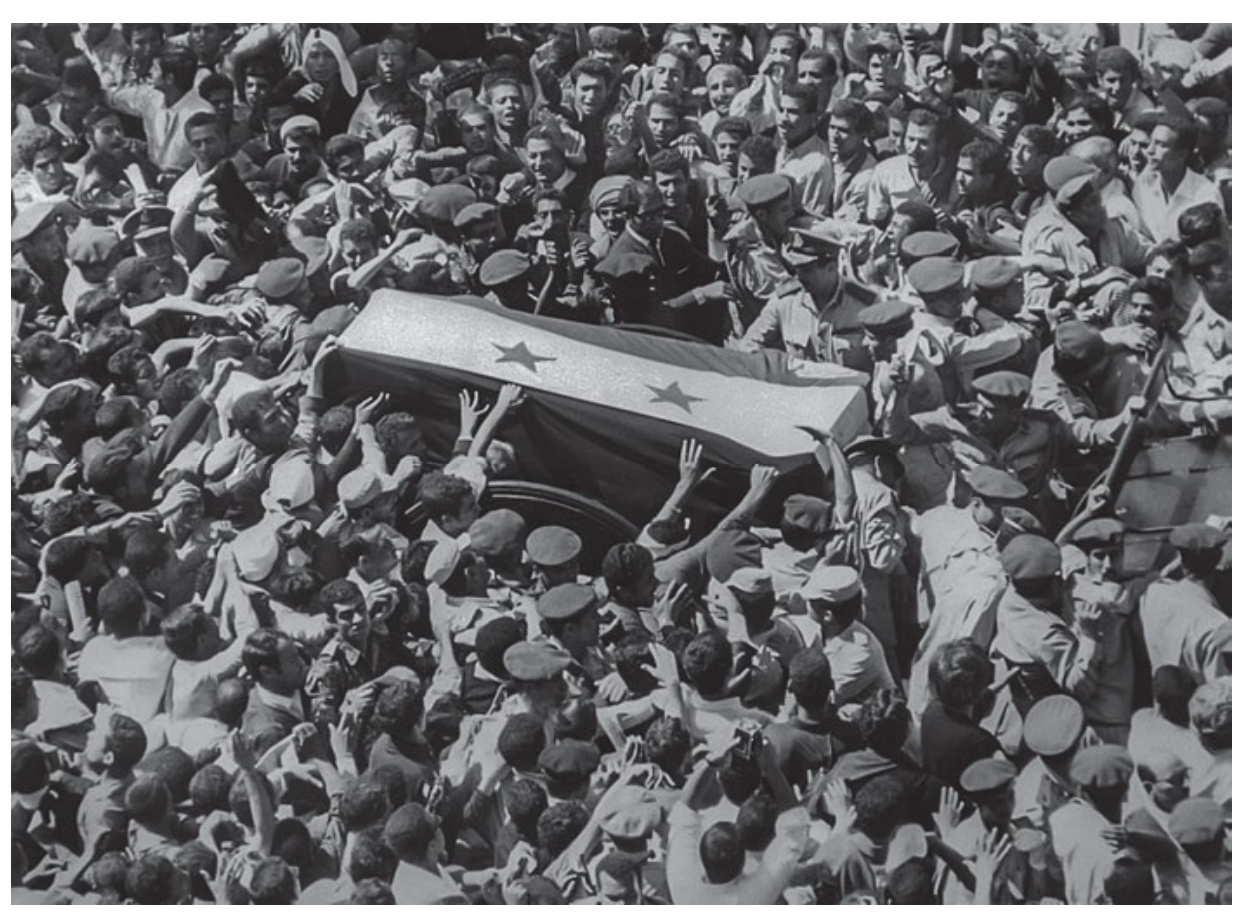

Abb. 2: Trauernde Menschenmasse um den Sarg Nassers.

wiegend in der Masse gestürzt und totgetreten worden waren. ${ }^{15}$ Wie auch am Tag von Nassers Tod und nach der Niederlage von 1967 gibt es keine Hinweise darauf, dass dieser Menschenansturm inszeniert gewesen sein könnte. Alles spricht dafür, dass es sich um ein spontanes, hoch emotionalisiertes Ereignis handelte, das die Verantwortlichen überraschte und überrumpelte. Wiederum beschworen die von der Menge skandierten Slogans in einer Mischung aus Trotz, Trauer und Verheißung das Weiterleben Nassers. Sie richteten sich auch gegen die anwesenden arabischen Führer, König Hussein von Jordanien und Yāsir 'Arafāt, mit denen Nasser unmittelbar vor seinem Herzinfarkt zur Beilegung der Staatskrise in Jordanien verhandelt hatte und denen die Trauernden die Schuld am Tod des Präsidenten zuwiesen: „Was habt ihr ihm angetan?" Schließlich griff die Menge den Slogan auf, der 1967 Nasser dazu gebracht hatte, seinen Rücktritt zurückzuziehen: „Gamal, du Licht unserer Augen, verlässt du die Araber? Wohin gehst du?"“16

15 Vgl. Der Spiegel: Nasser. Es kann nicht sein (Anm. 1); BBC: Mourners Killed as Nasser is Buried, 1. Oktober 1970, http://news.bbc.co.uk/onthisday/hi/dates/stories/october/1/news id_2485000/2485899.stm, 20. März 2018; Hofstadter: Egypt \& Nasser (Anm. 13), S. 262; Stephens: Nasser (Anm. 11), S. 557; Peter Woodward: Nasser, London 1992, S. 128.

16 Vgl. Aburish: Nasser (Anm. 12), S. 317 (in ägyptischem Arabisch: Yā Gamāl, yā nūr al-'ayn, sāyib al-'arab? Rāyiḥ 'alā fayn?); Vakitiotis: Nasser and His Generation (Anm. 10), S. 355. 
Der verstorbene Nasser wurde als Führer und Held besungen, ${ }^{17}$ doch sind in den Abgesängen auf ihn auch deutliche religiöse Anklänge zu erkennen: die Trauer über den Verlust einer Erlöserfigur und der Wunsch nach ihrem Weiterleben. Zahlreiche arabische Dichter widmeten ihm Nachrufe, von der palästinensischen Schriftstellerin Fadwā Țūqān, die über Nasser schrieb, der Wind habe sein Kommen vorausgesagt, bis zu der libanesischen Autorin Nağlā Abū 'Izz ad-Din, die darauf hinwies, dass Nasser am gleichen Tag wie der Prophet Muhammad gestorben sei. ${ }^{18}$ Der eminente palästinensische Dichter Mahmūd Darwiš gedachte Nassers in seinem Gedicht „Der Mann mit dem grünen Schatten“ (Ar-rağul d̦ū z-zill alabdar), in dem er zwar davor zurückschreckte, Nasser einen prophetischen Status zuzuschreiben, ihn aber doch zumindest als Abbild des Propheten darstellt:

Du bist kein Prophet

aber dein Schatten ist grün.

Wir leben mit dir

wir marschieren mit dir

wir hungern mit dir

und wenn du stirbst

versuchen wir, nicht mit dir zu sterben.

Auf deinem Grab wächst junges Korn

und frisches Wasser kommt herab

und du siehst uns

weitermarschieren

weitermarschieren

weitermarschieren. ${ }^{19}$

Der wohl berühmteste aller Nachrufe auf Nasser stammt von Nizār Qabbānī. Er greift die Klagerufe der Bevölkerung während der Beerdigung auf und beginnt mit den folgenden Worten, die in direktem Widerspruch zu Mahmūd Darwišs Zurückhaltung stehen: „Wir haben dich getötet, oh letzter der Propheten/ wir haben dich getötet." Es folgt ein Feuerwerk von historischen, politischen und religiösen Anspielungen, unter anderem auf das schiitische Motiv des Martyriums des Prophetenenkels Husain: „Alle unsere Tage sind Kerbela“. Qabbānī zieht schließlich folgendes Fazit:

Alle Mythen starben

mit deinem Tod, und Schehrazad hat Selbstmord begangen.

Hinter dem Begräbniszug zogen die Qurayš. ${ }^{20}[\ldots]$

Die dich verließen, ${ }^{21}$ haben sich versammelt

17 Vgl. al-Aḅbār: Faqadnā 'Abd an-Nāṣir. Māt az-za'ìm wa-l-qā’id wa-l-bațal, 29. September 1970, S. 1.

18 Vgl. Aburish: Nasser (Anm. 12), S. 316. Vgl. auch die Sammlung von Elegien auf http://

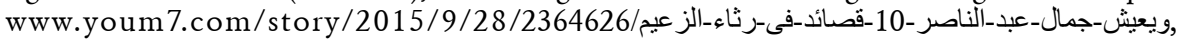
20. März 2018.

19 Maḥmūd Darwišs: Diwān Maḥmūd Darwišs, Bd. 1, Beirut ${ }^{14} 1996$, S. 361-364.

20 Der arabische Stamm, dem Muhammad angehörte.

21 Im Original: al-buawāriǧ, d. h. die Kharidschiten. 


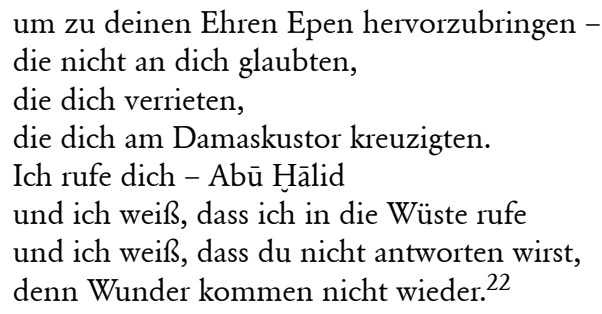

Was blieb, war eine überwältigende Nostalgie. Nağib Maḥfūz legte 1985 einem seiner Protagonisten die folgenden Worte in den Mund:

Ach, wo ist die gute, alte Zeit hin? Es gab wirklich schöne Tage, auch für mich. [...] Wir haben miteinander diskutiert, wir haben gelacht, das Studium machte Spaß, und es gab einen Mann, den man als Helden verehrte. Wir waren das Volk, und das Herz des Volkes hat ihn geliebt. Diese Liebe war ein Strauß herrlichster Rosen, den die schönsten Hoffnungen umhüllten und schützten. Wir verloren unseren ersten Präsidenten [...]. ${ }^{23}$

\section{Khomeini}

Die sakralen Dimensionen der Verehrung des Revolutionsführers Ayatollah Khomeini in Iran sind offensichtlicher und aufgrund des zentralen Islambezugs seiner politischen Botschaft auch nicht verwunderlich. Bereits im Verlauf der Jahre 1978/79 verfestigte sich sein Status als Revolutionär und Volksheld einerseits und als Referenzfigur für explizit schiitisch formulierte messianische Bedürfnisse und Hoffnungen andererseits. ${ }^{24}$ Khomeini gelang es, aus dem Exil heraus einen Zugriff auf die revolutionären Prozesse in Iran zu erlangen, der ihn zur Repräsentationsfigur nicht nur islamistischer Gruppierungen avancieren ließ, sondern darüber hinaus zum Gesicht der heterogenen Opposition gegen Mohammad Reza Shah erhob. ${ }^{25}$ In der Symbolik seiner Rückkehr nach Iran bei nahezu gleichzeitiger Flucht des Shahs ins Exil im Januar und Februar 1979 verdichtete sich demnach der Eindruck, dass Ayatollah Khomeini jener Held der iranischen Revolution sei, der den Niedergang der persischen Monarchie orchestrierte. ${ }^{26}$ Die nachhaltige historische Leistung des Revolutionsführers würde dabei jedoch erst in der erfolgreichen Implementierung eines politischen Systems in Iran zu sehen

22 Qabbāni: Al-a`māl as-siyāsiyya (Anm. 8), S. 353-364. Nizār Qabbānī schrieb nicht weniger als vier Elegien für Nasser, unter anderem Die vierte Pyramide. Sie enthalten durchweg nicht nur historische und politische Heroisierungsnarrative, sondern auch muslimische und christliche Motive des Prophetentums und Messianismus. Vgl. ebd., S. 365-390.

23 Nagib Machfus (Nağib Maḥuuzz): Der letzte Tag des Präsidenten. Übers. Doris Kilias, Zürich 2003, S. 31.

24 Vgl. Daniel Brumberg: Reinventing Khomeini. The Struggle for Reform in Iran, Chicago 2001, S. 96.

25 Vgl. Moin: Khomeini (Anm. 2), S. 176.

26 Vgl. Hamid Dabashi: Shi'cism. A Religion of Protest, Cambridge 2011, S. 273. 
sein, welches sich dezidiert als islamisch definiert und bis heute die verfassungsmäßige Grundlage der Islamischen Republik Iran darstellt. ${ }^{27}$

Nachdem sich im Mai 1989 sein Gesundheitszustand aufgrund eines Krebsleidens rapide verschlechterte, verstarb Khomeini mit (vermutlich) 87 Jahren in der Nacht vom 3. auf den 4. Juni. Er soll sich mit folgenden Worten von seiner Familie verabschiedet haben: „Es ist ein beschwerlicher Weg. Achtet auf all eure Worte und Taten. Ich habe nichts mehr hinzuzufügen. Wer bleiben will, kann bleiben. Wer gehen will, soll gehen. Löscht das Licht. Ich will schlafen. ${ }^{\text {"28 }}$ Die letzten Worte Ayatollah Khomeinis zitieren dabei nicht nur eine überlieferte Aussage des dritten Imams der schiitischen Glaubenslehre, al-Husayn b. 'Ali (626-680), bevor dieser in seiner letzten Schlacht bei Kerbala den Märtyrertod starb, sondern suggerieren auch eine Ruhe, die durch die Ereignisse der folgenden drei Tage konterkariert werden sollte; denn die Bestattung Ayatollah Khomeinis nahm einen Verlauf, welcher den Zeitzeugen und CBS-Reporter Tom Fenton später konstatieren ließ: „The authorities were trying to organize a state funeral, but it was like trying to organize a whirlwind. [...] Ayatollah Khomeini would be buried as he lived, in the eye of a religious storm. ${ }^{\text {29 }}$

Die Zusammenfassung der Ereignisse der folgenden drei Tage zeigt, dass die Wahrnehmung von einer Beerdigung, an der eine bestimmbare, wenn auch unvorstellbar große Menge von Menschen teilnahm, kaum haltbar ist. Der britische The Telegraph behauptet etwa, es handle sich um die viertgrößte Menschenansammlung der Geschichte und will ungefähr zehn Millionen Teilnehmer gezählt haben ${ }^{30}$ - eine Zahl, die freilich unmöglich zu belegen ist. Man muss eher von einem Land in Bewegung sprechen. Der Tod Khomeinis wurde mit seinem Bekanntwerden zum alles bestimmenden Thema der kommenden Tage, welches sich bis in die letzten Winkel Irans auszubreiten schien. Das Gravitationszentrum der Prozesse wurde dabei durch den Körper des Verstorbenen repräsentiert, auf den sich die Ereignisse gleichsam zubewegten.

Obwohl man im inneren Zirkel der Macht beschlossen hatte, den Tod Khomeinis erst im Laufe des 4. Juni bekanntzugeben, nachdem man sich auf einen Nachfolger geeinigt hatte, verbreitete sich die Nachricht vom Ableben des Revolutionsführers mit rasender Geschwindigkeit. Khomeini war bereits zwei Wochen zuvor in das Krankenhaus im Norden Teherans eingeliefert worden und verbrachte seine letzten Tage durchaus öffentlich. Sein Gebrechen und sein nahendes Ende waren

27 Vgl. Vanessa Martin: Creating an Islamic State. Khomeini and the Making of a New Iran, London/New York 2003.

28 Moin: Khomeini (Anm. 2), S. 227. Siehe auch Michael Axworthy: Revolutionary Iran. A History of the Islamic Republic, London 2013, S. 304.

29 Tom Fenton: The Day they Buried the Ayatollah, in: Iranian Studies 41, 2008, S. 242.

30 Vgl. Alice Philipson: The Ten Largest Gatherings in Human History, in: The Telegraph, 19. Januar 2017, http://www.telegraph.co.uk/news/newstopics/howaboutthat/11354116/The -ten-largest-gatherings-in-human-history.html, 18. Mai 2018. 
Teil der täglichen medialen Berichterstattung. ${ }^{31}$ Man wartete daher auf den Tod Khomeinis, und als sich die ersten Gerüchte über sein Ableben verbreiteten, veränderte sich die Stimmung im Land. Mit dem Sonnenaufgang setzte sich im wichtigen Medium des Radios ein düsterer und pathetischer Ton durch. Normale Radiosendungen wurden abgebrochen und durch Koranrezitationen ersetzt. Um 7 Uhr verkündete dann ein weinender Moderator den Tod Khomeinis. ${ }^{32}$ Hierauf setzte sich nun eine landesweite Massenbewegung in Gang, die an der noch gar nicht terminierten Bestattung des Ayatollah teilhaben wollte. ${ }^{33}$

Der verstorbene Revolutionsführer wurde derweil gemäß den islamischen Vorschriften gewaschen, gekleidet und in Blickrichtung Mekka aufgebahrt. Am 5. und 6. Juni war er öffentlich in einem gläsernen und gekühlten Sarg in Teheran zu sehen. Parallel zu den politischen Entscheidungsprozessen, die der Tod des Nationalhelden notwendig machte und die sich um sein politisches Erbe drehten, ${ }^{34}$ zeigte sich bereits am 5. Juni, dass die Bedürfnisse der Masse auf den Körper des Verstorbenen gerichtet waren und sich die Menge im Angesicht der Leiche Khomeinis kaum kontrollieren lassen würde. Gleichwohl bemühte man sich um einen würdevollen Abschied. In der Sommerhitze wurde die Feuerwehr eingesetzt, um die Menschenmenge mit Wasser zu kühlen, ${ }^{35}$ die Revolutionsgarden versuchten den Körper zu bewachen und die schiitische Geistlichkeit war um die Einhaltung der islamischen Rituale bemüht, sodass es tatsächlich zumindest zu einem Moment der Ruhe kam: Am Morgen des 6. Juni wurde der Körper Khomeinis auf Anweisung Großayatollah Golpayeganis aus dem gläsernen Sarkophag gehoben, damit dieser die entsprechenden Gebete verrichten konnte. Der Körper wurde in einem offenen Sarg gleichsam in die Menge hineingehoben, wo Golpayegani die Gebete sprach, und für etwa zwanzig Minuten standen die Ereignisse still. ${ }^{36}$

Danach jedoch eskalierte die Situation: ${ }^{37}$ Eine Prozession sollte den Körper zum $20 \mathrm{~km}$ südlich von Teheran gelegenen Märtyrerfriedhof geleiten, gelangte aber nicht weit. Der Leichenwagen ging schlicht verloren in den schieren Menschenmassen, die vom Streben geprägt schienen, an den Körper des Ayatollahs zu gelangen. ${ }^{38}$ Revolutionsgarden mussten die Hoheit über den Leichenwagen zurückerobern, sodass Schüsse in die Luft gefeuert wurden und die Wasserschläu-

\footnotetext{
Siehe nur https://www.youtube.com/watch?v=KiRq-J-Bhl4, 16. Mai 2018.

https://www.youtube.com/watch?v=eJEbnjLqjVE, 16. Mai 2018.

Vgl. Moin: Khomeini (Anm. 2), S. 304.

Gumbūri-ye Eslami, 16. Hordād 1368 [6. Juni 1989].

Vgl. Axworthy: Revolutionary Iran (Anm. 28), S. 304.

Vgl. Moin: Khomeini (Anm. 2), S. 311.

37 Die Rekonstruktion der Ereignisse erfolgt auf Grundlage der Berichte von Fenton: The Day they Buried the Ayatollah (Anm. 29); Moin: Khomeini (Anm. 2), S. 311-312; sowie frei zugänglichem Filmmaterial (hier insbesondere: http://emam.com/posts/view/مر اسم تنييع-جنازه-امام, 16. Mai 2018).

38 Vgl. Fenton: The Day they Buried the Ayatollah (Anm. 29), S. 243.
} 
che der Feuerwehr nun zur Disziplinierung der Massen eingesetzt wurden. ${ }^{39}$ Das Fernsehen stoppte die Liveübertragung, um einen weiteren Zustrom zu verhindern, und nachdem die Revolutionsgarden die Leiche Khomeinis vor den Massen in Sicherheit gebracht hatten, wurde offiziell bekanntgegeben, dass die Bestattung verschoben worden sei - eine bewusste Falschmeldung, um der Lage Herr werden zu können. Dies hielt die Massen jedoch nicht davon ab, sich in Richtung des Friedhofs zu bewegen, wo sie die Beisetzung Khomeinis erwarteten. Fenton berichtet über seinen Eindruck aus dem Hubschrauber: „Once we were airborne, I was stunned by what I saw. Great black rivers of mourners were pouring out of the city, all flowing toward the area where we were heading. There were clearly millions. None of us could estimate the number." 40

Khomeinis Leiche wurde derweil ebenfalls mit einem Hubschrauber zum Friedhof gebracht. Mit dem Moment der Landung jedoch verlor man erneut die Kontrolle über die Situation. Die Menge strömte umgehend wieder auf den verstorbenen Ayatollah zu, der in einem offenen Sarg aus dem Hubschrauber gehoben wurde. ${ }^{41}$ Es gelang zwar, den Sarg samt Leichnam auf einen Krankenwagen zu heben, mit welchem dieser zum vorgesehenen Grab verbracht werden sollte; die Ekstase der Menge führte jedoch schließlich dazu, dass der Körper aus dem Sarg gezogen wurde. ${ }^{42}$ Erneut musste der Körper Khomeinis vor den Massen ,gerettet' werden und sollte mit dem Hubschrauber weggeschafft werden.

Als es schließlich gelang, den Leichnam zurück in den Hubschrauber zu bringen, drängte die Menschenmenge auf den Helikopter zu, der in einer Meeresflut zu versinken schien. Ungeachtet dessen versuchte der Pilot, aus der Menschenmenge heraus zu starten: „Dozens of persons were clinging to the aircraft, refusing to let it go as the rotors revved up. It was touch-and-go for a few moments until the helicopter finally broke free, soaring into the sky with the open coffin precariously half hanging out of an open door." ${ }^{\text {"43 }}$ Es gelang erst gegen Abend, Khomeini in einem Metallsarg zur Grabungsstätte zu schaffen, über die eiligst ein Container gestellt wurde, um das Grab zu sichern: jener Ort, auf dem später ein Grabmal errichtet wurde, das als nationales und religiöses Symbol die Geschichte der Islamischen Republik Iran gleichsam an Khomeinis statt repräsentieren soll. ${ }^{44}$ Nicht erst seit seinem Tode 1989, sicher aber auch bedingt durch die Wucht der Ereignisse um seine Beisetzung und die damit verbundene Botschaft, dass die Massen dem Revolutionsführer ihre Verehrung trotz aller Krisen zollten, ist das Bild Ayatollah Khomeinis als Gründungsvaters der Islamischen Republik Iran bis

39 Vgl. Moin: Khomeini (Anm. 2), S. 311.

Fenton: The Day they Buried the Ayatollah (Anm. 29), S. 243.

Ein Videomitschnitt dieser Szene online abrufbar unter: http://emam.com/posts/view/ مر اسم-تشييع-جناز هـامام, Min. 00:03:50-00:04:35.

Vgl. Fenton: The Day they Buried the Ayatollah (Anm. 29), S. 243.

Ebd., S. 244.

Vgl. Kishwar Rizvi: Religious Icon and National Symbol. The Tomb of Ayatollah Khomeini in Iran, in: Muqarnas 20, 2003, S. 209. 
heute allgegenwärtig. Als Symbol für die Nation selbst ist es Teil seiner ständigen medialen Präsenz im öffentlichen Raum.

\section{Sterblichkeit und Heiligkeit}

Die religiösen Anklänge in den Reaktionen der Bevölkerungen Ägyptens und Irans auf den Tod von als übermächtig wahrgenommenen Führerfiguren sind unübersehbar. Für Khomeini, den Großayatollah, mag dies auf der Hand liegen; tatsächlich hatte das Regime aber Versuche, ihm einen messianischen oder gar göttlichen Status zuzusprechen, dezidiert zurückgewiesen. ${ }^{45}$ Nasser wiederum repräsentierte das Primat ,fortschrittlicher ${ }^{r}$ arabischer Politik über die Religion. Dennoch erfahren beide Figuren im Angesicht ihres Todes eine Verehrung, die sie ins Sakrale überhöht und Erlösungssehnsüchte auf sie projiziert. Und vielleicht ist es gerade ihr Tod, der dies ermöglicht - im Falle Khomeinis abzusehen, im Falle Nassers unerwartet. Beide Figuren ereilte der Tod, nachdem die durch sie repräsentierten Regime tiefe Krisen durchlaufen hatten. Die Niederlage Ägyptens im Sechs-Tage-Krieg von 1967 hatte Nasser schwer beschädigt; der verlustreiche Krieg Irans gegen den Irak von 1980 bis 1988 war kaum mehr geeignet, Khomeinis charismatischen Führungsanspruch zu legitimieren.

Im Angesicht des Todes verblassten jedoch Krisen, Fehlentscheidungen und Niederlagen. Ihr Tod ermöglichte es, die beiden Nationalhelden als vollkommene Symbole einer vergangenen, größeren Zeit zu überhöhen und zu verehren, als Bollwerke gegen eine ungewisse Zukunft, der - und dies bedingte maßgeblich die affektive Eruption der Ereignisse - ihre Anhänger nun, nach ihrem Dahinscheiden, schutzlos ausgeliefert schienen. Alle zukünftigen Krisen und Fehler sollten auf das Konto ihrer schwächeren, fehlbareren, menschlicheren Nachfolger gehen; alle Ängste vor einem Wiedererstarken übermächtiger Antagonisten sollten auf diese projiziert werden. Ein verstorbener Führer macht keine Fehler mehr; der verstorbene Held kann sein Charisma nicht mehr aufs Spiel setzen, sein Nimbus in den Routinen des Alltags nicht veralltäglicht werden. ${ }^{46}$ Zugleich liegt im Tod eine ambivalente Botschaft: Einerseits erlangt der Held im Tod seine Vollkommenheit, die ihm eine sakrale Dimension verleiht - eine Dimension, die sich in der Verehrung der Massen, den überwältigenden Ausmaßen seiner Beerdigungsfeier, widerspiegelt oder gar erst durch sie konstituiert wird. Andererseits beweist der Tod des Verehrten sein Menschsein. Er ist eben kein Gott, sondern nur der Held seiner Gemeinde, nur Repräsentant einer Epoche. Im Umkehrschluss verfestigt sich das Bild, dass die Verfasstheit der Gegenwart auf ebenso tönernen Füßen steht wie das Leben desjenigen, der diese Gegenwart repräsentiert.

45 Vgl. Olmo Gölz: Khomeini's Face is in the Moon. Limitations of Sacredness and the Origins of Sovereignty, in: Felix Heinzer u. a. (Hg.): Sakralität und Heldentum (Helden Heroisierungen - Heroismen 6), Würzburg 2017, S. 229-244.

46 Vgl. Bernhard Giesen: Triumph and Trauma, Colorado 2004, S. 19. 


\section{Heldentod und Epochenbruch}

Die hoch emotionalisierten Prozesse um die Beisetzungen Nassers und Khomeinis und die Versuche der Massen, sich der Leichname der beiden Nationalhelden zu bemächtigen, sind nur vor dem Hintergrund der historischen Dimensionen zu verstehen, die in beiden Akteuren repräsentiert werden. Die Bedeutung der beiden herausragenden Figuren der Geschichte des Nahen und Mittleren Ostens im 20. Jahrhundert lässt sich dabei an zahlreichen Indikatoren ermitteln, auf die eingangs bereits hingewiesen wurde: Der Militärputsch der Gruppe der ,Freien Offiziere' unter der Führung Nassers von 1952 führte nicht nur zur Abschaffung der ägyptischen Monarchie und zur Gründung einer Republik, sondern hatte eine dezidiert anti-imperialistische Stoßrichtung, ${ }^{47}$ die Ägypten einen prominenten Platz in der Dekolonisierungsbewegung verschaffte. Der Status Nassers als Nationalheld ist daher auch über die erfolgreiche Machtprobe mit seinem eigentlich als übermächtig wahrgenommenen Antagonisten zu erklären, nämlich dem westlichen Imperialismus. Auf einer ähnlichen Logik - angereichert mit einer explizit islamischen Rhetorik - gründete sich auch der Status Ayatollah Khomeinis als populärer Führer im Angesicht des übermächtigen Westens. Bereits 1980 konstatiert Roy Mottahadeh: „Khomeini is a new kind of leader in the modern Islamic Middle East. Like Nasser, he draws part of his popular strength from his ability to defy Western governments that had previously been believed to have coercive powers over Middle Eastern countries. “48 Khomeini gelang es zudem, einen Diskurs zu prägen, der die Welt in ,Unterdrücker ${ }^{\varsigma}$ und ,Unterdrückte' unterteilte und so einen dauernden Kampf des Bösen gegen das Gute zu konstruieren, in dem das Böse zwar übermächtig schien, wohl aber von seiner eigenen Bewegung in die Schranken gewiesen werden konnte. ${ }^{49}$

Sowohl der ägyptische Militärputsch von 1952 als auch die Iranische Revolution von 1979, die von der Mehrheit der Bevölkerungen der jeweiligen Länder explizit als die historischen Leistungen von Nasser und Khomeini interpretiert wurden, hatten aufgrund dieser ideologischen Komponenten Auswirkungen über die Staatsgrenzen Ägypten und Irans hinaus, sodass im ersten Fall der arabische Nationalismus die arabische Welt nachhaltig prägte, wohingegen durch das Beispiel Irans der politische Islam an Bedeutung gewann. Die Umstürze hatten einen profunden Einfluss auf das Staatensystem des Nahen und Mittleren Ostens, ${ }^{50}$ woraus sich auf der Wahrnehmungsebene zwei konfligierende Effekte ableiten lassen: Einerseits wurden durch die erfolgreichen Systemwechsel in beiden Fällen mit der

47

48

49

Vgl. Misagh Parsa: Social Origins of the Iranian Revolution, New Brunswick 1989, S. 2; Rob Johnson: Mustazafin and taghutti. Iran and the War, 1980-1988, S. 56-74, in: Nigel John Ashton / Bryan R. Gibson (Hg.): The Iran-Iraq War. New International Perspectives, London/New York 2013.

50 Vgl. Nahas: State Systems and Revolutionary Challenge (Anm. 3), S. 507. 
neuen Ordnung Hoffnungen auf eine bessere Epoche verbunden; andererseits war im Angesicht der lokalen wie globalen Kräfte, die aufgrund der internationalen Dimensionen auf diese Ordnung wirkten, zumindest die Angst vor einer Destabilisierung zu spüren. Diese Unsicherheit verknüpfte sich insbesondere mit der Frage, was passieren würde, wenn die ordnungsstiftenden Paradigmen wegbrechen oder scheitern sollten. In den Personen Nasser und Khomeini sowie später im Angesicht ihres Todes verdichteten sich diese Hoffnungen und Ängste axiomatisch. Beide wurden nicht nur als Repräsentanten jener Ordnungen gesehen, die sie selbst geschaffen hatten, sondern aufgrund ihrer eigenen Leistung - insbesondere im Angesicht ihrer historisch übermächtig erscheinenden Antagonisten auch als einzige Garanten dieser neuen Ordnung eingeschätzt.

Hierauf begründete sich der Heldenstatus Nassers und Khomeinis. Dieser Status - und auch das wird in den hier geschilderten Prozessen klar - ist deutlich von Heroisierungsmodellen abzugrenzen, die etwa auf vorbildliches Handeln oder ertragenes Leid des als Helden verehrten Protagonisten rekurrieren, wie dies zum Beispiel für die Idee des Kämpfers oder des gerade für den schiitischen Kontext so zentralen Märtyrers der Fall ist. Der Heldenstatus Nassers und Khomeinis lässt im Gegensatz dazu keine Nachahmbarkeit zu, sondern verweist auf die Einzigartigkeit ihrer Handlungen. Beide Protagonisten wurden von den Massen zweifellos als jene „welthistorischen Individuen“ empfunden, die aus einer Quelle schöpfen, deren Geist, wie Hegel formuliert „an die Außenwelt wie an die Schale pocht und sie sprengt" und deren Taten Zustände und Weltverhältnisse zu evozieren scheinen, die als ihr eigenes Werk empfunden werden müssen. ${ }^{51}$ Beide schienen somit für die Systeme verantwortlich, die sie implementierten, und wurden als die Heldenfiguren verehrt, die sich nicht an die überkommenen Regeln - Hegels ,Schale der Außenwelt ${ }^{6}$ - halten mochten, sondern deren souveränes Handeln neue Regeln kreierte und die Grundlagen für eine neue Ordnung, ibre Ordnung, legte. ${ }^{52} \mathrm{Im}$ Angesicht des Umgangs der Massen mit dem Tod der beiden Nationalhelden wird genau diese Auffassung deutlich - und damit auch die Angst vor der ungewissen Zukunft, die das Wegbrechen der Garanten der Ordnung erwarten lässt. Die Aneignung der Leichname durch die Massen ist als symbolischer Versuch zu werten, sich des nun wie sicher scheinenden Epochenbruchs zu erwehren. Es geht nicht nur um eine religiös aufgeladene Berührung. Die Menschen hängen sich an den startenden Helikopter, die Masse versperrt den Weg zum Grab: Wie in der Zuspitzung aller Symbolik wird dem Helden, dem „Besten der Zeit“",53 verboten zu gehen, denn ohne ihn ist die Zukunft ungewiss.

51 Hegel: Philosophie der Geschichte (Anm. 6), S. 45-46.

52 Vgl. Giesen: Triumph and Trauma (Anm. 46), S. 2: „The charismatic hero is presented as a mediator between the realm of the sacred, but he is also exempted from the constraints of regular rules. Instead, his sovereign action creates new rules and lays the ground for a new order."

53 Hegel: Philosophie der Geschichte (Anm. 6), S. 46. 


\section{Abbildungsnacbreise}

Abb. 1: Mériam Belli: An Incurable Past. Nasser's Egypt Then and Now. Gainesville, FL: University of Florida Press, 2013, S. 125.

Abb. 2: https://www.gettyimages.de/detail/nachrichtenfoto/mourners-reach-outto-touch-the-flag-draped-coffin-of-nachrichtenfoto/515398650. 
